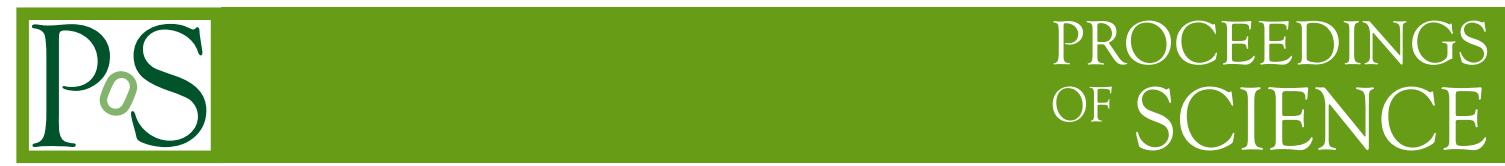

Measurements of $\left|V_{c b}\right|$

\title{
Christoph Schwanda*
}

HEPHY, Austria

E-mail: Schwanda@hephy.oeaw.ac.at

In this article, we discuss the status of the determination of the Cabibbo-Kobayashi-Maskawa matrix element $\left|V_{c b}\right|$ from semileptonic $B$ decays. We review measurements based on inclusive and exclusive $B$ decays and give the winter 2009 world average numbers for $\left|V_{c b}\right|$ obtained using these two approaches.

Flavor Physics and CP Violation 2009

May 27-June 12009

Lake Placid, NY, USA

${ }^{*}$ Speaker. 


\section{Introduction}

The magnitude of the Cabibbo-Kobayashi-Maskawa (CKM) matrix element $\left|V_{c b}\right|$ [1] can be determined from semileptonic $B$ decays to charmed final states. There are determinations using all semileptonic final states in a given region of phase space ("inclusive measurements"), and analyses based on specific final states only ("exclusive measurements"). As the theoretical and experimental uncertainties are different for both approaches, consistency between inclusive and exclusive is an important cross-check on our understanding.

The theory of the determination of $\left|V_{c b}\right|$ from inclusive and exclusive decays is discussed elsewhere [2]. In this article, first we briefly explain how to measure semileptonic $B$ decays at the $B$ factory experiments Belle [3] and BaBar [ $₫$ ]. Then, recent analyses of inclusive and exclusive $B$ decays, mostly at the $B$ factories, are reviewed and the world average values of $\left|V_{c b}\right|$ are given.

\section{Measuring semileptonic $B$ decays}

Belle and BaBar have both recorded large samples of $e^{+} e^{-}$collisions at the center-of-mass (c.m.) energy of the $\Upsilon(4 S)$ resonance, containing several hundreds of millions of $B \bar{B}$ events. At this energy, a pair of $B \bar{B}$ mesons is produced at threshold, leading to special kinematics that simplify the reconstruction of semileptonic $B$ meson decays in particular:

First, as no fragmentation particles are produced, the $B$ meson energy in the c.m. frame is equal to the beam energy $E_{\text {beam }}$. This allows to define two nearly independent variables for selecting $B$ signals, the beam-constrained mass $M_{\mathrm{bc}}=\sqrt{E_{\mathrm{beam}}^{2}-\vec{p}_{B}^{2}}$ and the energy difference $\Delta E=E_{B}-E_{\text {beam }}$, where $\vec{p}_{B}$ and $E_{B}$ are the $B$ candidate 3-momentum and energy in the c.m. frame, respectively. Then, by reconstructing one $B$ meson in the event, the 4-momentum of the other $B$ is automatically known, even if the final state of the other $B$ decay contains undetected particles, such as a neutrino. Finally, in the c.m. frame, $B \bar{B}$ events are spherical while other hadronic events $e^{+} e^{-} \rightarrow q \bar{q}, q=u, d, s, c$, are more jet-like. This allows to reject hadronic continuum by placing requirements on event shape variables.

A technique commonly used in the analysis of semileptonic $B$ decays is tagging, i.e., the full or partial reconstruction of the non-signal $B$ meson in the event to reduce backgrounds or obtain information on the signal $B$. The most extreme form of tagging is the full-reconstruction tag, where the hadronic decay of one $B$ meson in the event is fully reconstructed. This allows to determine the 4-momentum of the signal $B$ meson and to virtually eliminate combinatorial background, as all particles in the event stemming from the decay of the other $B$ can be identified. Of course, a fullreconstruction tagged analysis is only feasable for very large data samples, as the tagging efficiency is of $\mathscr{O}\left(10^{-3}\right)$. The opposite approach, i.e., making no requirements on the non-signal $B$ is known as untagged analysis. This strategy yields the largest amount of signal events but obviously suffers from combinatorial backgrounds and associated systematic uncertainties.

\section{3. $\left|V_{c b}\right|$ from inclusive semileptonic $B$ decays to charm}

\subsection{Theoretical framework}

To obtain the CKM matrix element $\left|V_{c b}\right|$ from inclusive semileptonic $B$ decays $B \rightarrow X_{c} \ell v$, one 
Table 1: Non-perturbative parameters in the expressions derived in the kinetic and 1S schemes.

\begin{tabular}{c|c|c}
\hline \hline & kinetic scheme & 1 S scheme \\
\hline $\mathscr{O}(1)$ & $m_{b}^{\text {kin }}, m_{c}^{\text {kin }}$ & $m_{b}^{1 S}$ \\
$\mathscr{O}\left(1 / m_{b}^{2}\right)$ & $\mu_{\pi}^{2}, \mu_{G}^{2}$ & $\lambda_{1}, \lambda_{2}$ \\
$\mathscr{O}\left(1 / m_{b}^{3}\right)$ & $\rho_{D}^{3}, \rho_{L S}^{3}$ & $\rho_{1}, \tau_{1}, \tau_{2}, \tau_{3}$ \\
\hline \hline
\end{tabular}

Table 2: Moments used in the HFAG global fit in the kinetic scheme. $n$ is the order of the moment. In total, there are 27 measurements from BaBar, 25 measurements from Belle and 12 from other experiments.

\begin{tabular}{l|ccc}
\hline \hline Experiment & $\left\langle M_{X}^{2 n}\right\rangle$ & $\left\langle E_{\ell}^{n}\right\rangle$ & $\left\langle E_{\gamma}^{n}\right\rangle$ \\
\hline BaBar & $n=1,2$ [11] & $n=0,1,2,3$ [12] & $n=1,2$ [13, 14] \\
Belle & $n=1,2$ [15] & $n=0,1,2,3$ [16] & $n=1,2$ [17] \\
CDF & $n=1,2$ [18] & & \\
CLEO & $n=1,2$ [19] & & $n=1$ [20] \\
DELPHI & $n=1,2$ [21] & $n=1,2,3$ [21] & \\
\hline \hline
\end{tabular}

uses calculations of the semileptonic $B$ decay width based on the Heavy Quark Effective Theory (HQET) and the Operator Product Expansion (OPE) [1] 6]. These predictions depend on $\left|V_{c b}\right|$ and a number of non-perturbative quantities such as heavy quark masses and expectation values of local operators. The non-perturbative parameters can be determined experimentally through measurements of other inclusive observables in $B$ decays such as the lepton energy $E_{\ell}$ and the hadronic mass $M_{X}^{2}$ moments in $B \rightarrow X_{c} \ell v$ and the photon energy $E_{\gamma}$ moments in $B \rightarrow X_{s} \gamma$ [5, 7, 8]. The moments are measured as a function of the minimum lepton or photon energy. In practice, $\left|V_{c b}\right|$ and the non-perturbative parameters are determined simultaneously from a global fit to all relevant experimental data.

There are two independent calculations of the semileptonic branching fraction and of the moments in $B \rightarrow X_{c} \ell v$ and $B \rightarrow X_{s} \gamma$ transitions that allow to perform this global analysis of inclusive $B$ decays. Refering to the $b$-quark mass definition used, the two sets of expressions are called 1S [5] and kinetic scheme [6, 7, 8] calculations. In both frameworks, non-perturbative corrections have been evaluated to the $\mathscr{O}\left(1 / m_{b}^{3}\right)$, Table 1 .

\subsection{Measurements of inclusive $B$ decays}

The Heavy Flavor Averaging Group (HFAG) [9] has combined data from Belle, BaBar and other experiments and performed a global fit in the kinetic scheme using a total of 64 measurements. The moments used are shown in Table 2 . In the following, we briefly review some of the analyses quoted in this table:

$\mathrm{BaBar}$ has updated their measurement of the hadronic mass moments $\left\langle M_{X}^{2 n}\right\rangle[10]$ and obtained preliminary results based on a dataset of $210 \mathrm{fb}^{-1}$ taken at the $\Upsilon(4 S)$ resonance [11]. In this analysis, the hadronic decay of one $B$ meson in $\Upsilon(4 S) \rightarrow B \bar{B}$ is fully reconstructed $\left(B_{\text {tag }}\right)$ and the 
semileptonic decay of the second $B$ is infered from the presence of an identified lepton ( $e$ or $\mu$ ) within the remaining particles in the event $\left(B_{\text {sig }}\right)$. The use of the full-reconstruction tag allows to significantly reduce combinatorial backgrounds and select semileptonic decays with a purity of about $80 \%$. Particles used neither for the reconstruction of $B_{\text {tag }}$ nor for the charged lepton are considered to belong to the $X_{c}$ system, and the hadronic mass spectrum $M_{X}$ is calculated using some kinematic constraints.

From this spectrum, BaBar calculates the hadronic mass moments $\left\langle M_{X}^{n}\right\rangle, n=1, \ldots, 6$ for minimum lepton momenta in the c.m. frame ranging from 0.8 to $1.9 \mathrm{GeV} / c$. These moments are however distorted by acceptance and finite resolution effects and an event-by-event correction is derived from Monte Carlo (MC) simulated events. These corrections are linear functions with coefficients depending on the lepton momentum, the multiplicity of the $X_{c}$ system and $E_{\text {miss }}-c\left|\vec{p}_{\text {miss }}\right|$, where $E_{\text {miss }}$ and $\vec{p}_{\text {miss }}$ are the missing energy and 3-momentum in the event, respectively. Note that this analysis measures also mixed mass and c.m. energy moments $\left\langle N_{X}^{2 n}\right\rangle, n=1,2,3$, with $N_{X}=M_{X}^{2} c^{4}-2 \tilde{\Lambda} E_{X}+\tilde{\Lambda}^{2}$ and $\tilde{\Lambda}=0.65 \mathrm{GeV}$ in addition to ordinary hadronic mass moments. These mixed moments are expected to better constrain some non-perturbative parameters though they are not used in current global fit analyses yet.

Belle has recently measured the hadronic mass [15] and c.m. electron energy [16] spectra in $B \rightarrow X_{c} \ell v$ decays, based on $140 \mathrm{fb}^{-1}$ of $\Upsilon(4 S)$ data. The experimental procedure is very similar to the $\mathrm{BaBar}$ analysis, i.e., one $B$ meson in the event is fully reconstructed in a hadronic channel. The main difference to the analysis discussed above is that detector effects in the spectra are removed by unfolding using the Singular Value Decomposition (SVD) algorithm [22] with a detector response matrix found from MC simulation. The moments are then calculated from the unfolded spectra. The hadronic mass analysis [15] measures the first and second moments of $M_{X}^{2}$ for minimum c.m. lepton energies between 0.7 and $1.9 \mathrm{GeV}$.

In Ref. [16], Belle measures the partial semileptonic branching fraction and the c.m. electron energy moments $\left\langle E_{e}^{n}\right\rangle, n=1, \ldots, 4$, for minimum c.m. electron energies ranging from 0.4 to $2.0 \mathrm{GeV}$.

An interesting analysis of inclusive $B \rightarrow X_{c} \ell v$ decays is done by the DELPHI experiment [21]: In this study, the $b$-frame lepton energy $\left\langle E_{l}^{n}\right\rangle, n=1,2,3$, and the hadronic mass $M_{X}^{2 n}, n=1, \ldots, 5$, moments are measured without applying any selection on the lepton energy in the $b$-frame. This is possible as DELPHI studies $b$-decays in $Z \rightarrow b \bar{b}$ events. $b$-hadrons are thus produced with significant kinetic energy in the laboratory frame, so that charged leptons at rest in the $b$-frame can be seen in the detector.

\subsection{Results of the global fit}

The HFAG global fit follows closely the procedure described in Ref. [23]. The results in terms of $\left|V_{c b}\right|, m_{b}$ and $\mu_{\pi}^{2}$ are quoted in Table 3 and Fig. 1. where $\mu_{\pi}^{2}$ is one of the non-perturbative parameters describing the motion of the $b$-quark inside the $B$ hadron. The total uncertainty in $\left|V_{c b}\right|$ is $1.7 \%$ with $1.0 \%$ due to the global fit, $0.2 \%$ from the $B$ lifetime and $1.4 \%$ theoretical error in the OPE expression of the semileptonic width [6]. Recently, concerns have been raised about the inclusion of $B \rightarrow X_{s} \gamma$ moments, as their prediction is not based on pure OPE but involves modeling of non-OPE contributions using a shape function. We therefore also quote the results of a fit including the $B \rightarrow X_{c} \ell v$ data only (53 measurements). 
Table 3: Results of the HFAG fit. In the last column we quote the $\chi^{2}$ of the fit over the number of degrees of freedom.

\begin{tabular}{l|cccc}
\hline \hline Dataset & $\left|V_{c b}\right|\left(10^{-3}\right)$ & $m_{b}(\mathrm{GeV})$ & $\mu_{\pi}^{2}\left(\mathrm{GeV}^{2}\right)$ & $\chi^{2} /$ ndf. \\
\hline$X_{c} \ell \nu$ and $X_{s} \gamma$ & $41.67 \pm 0.73$ & $4.601 \pm 0.034$ & $0.440 \pm 0.040$ & $29.7 /(64-7)$ \\
$X_{c} \ell \nu$ only & $41.48 \pm 0.75$ & $4.659 \pm 0.049$ & $0.428 \pm 0.044$ & $24.1 /(53-7)$ \\
\hline \hline
\end{tabular}
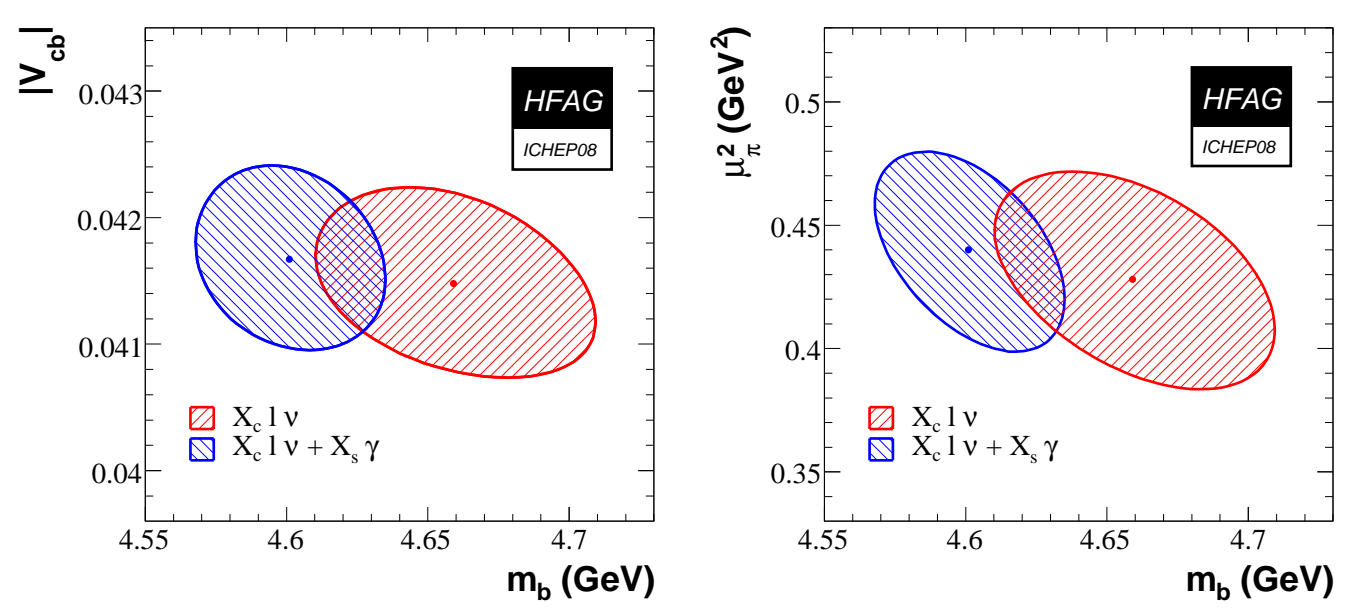

Figure 1: $\Delta \chi^{2}=1$ contours for the HFAG fit for $\left|V_{c b}\right|$ and $m_{b}$ in the $\left(m_{b},\left|V_{c b}\right|\right)$ and $\left(m_{b}, \mu_{\pi}^{2}\right)$ planes, with and without $B \rightarrow X_{s} \gamma$ data.

\section{4. $\left|V_{c b}\right|$ from exclusive semileptonic $B$ decays to charm}

\subsection{Differential decay rate}

The extraction of $\left|V_{c b}\right|$ from the exclusive decays $B \rightarrow D^{(*)} \ell v_{\ell}$ is based on the following expressions of the differential decay rates as a function of $w=v \cdot v^{\prime}$, where $v$ and $v^{\prime}$ are the 4-velocities of the initial and final state hadron in the decay [24],

$$
\begin{gathered}
\frac{d \Gamma}{d w}\left(B \rightarrow D^{*} \ell v_{\ell}\right)= \\
\frac{G_{F}^{2}}{48 \pi^{3}}\left|V_{c b}\right|^{2}\left(m_{B}-m_{D^{*}}\right)^{2} m_{D^{*}}^{3}\left(w^{2}-1\right)^{1 / 2}(w+1)^{2} \times\left[1+\frac{4 w}{w+1} \frac{m_{B}^{2}+m_{D^{*}}^{2}-2 w m_{B} m_{D^{*}}}{\left(m_{B}-m_{D^{*}}\right)^{2}}\right] \mathscr{F}^{2}(w), \\
\frac{d \Gamma}{d w}\left(B \rightarrow D \ell v_{\ell}\right)=\frac{G_{F}^{2}}{48 \pi^{3}}\left|V_{c b}\right|^{2}\left(m_{B}+m_{D}\right)^{2} m_{D}^{3}\left(w^{2}-1\right)^{3 / 2} \mathscr{G}^{2}(w) .
\end{gathered}
$$

Here, $m_{D}, m_{D^{*}}$ and $m_{B}$ are the $D, D^{*}$ and $B$ meson masses, respectively. In the limit of infinite heavy quark masses, heavy quark symmetry predicts the normalization of the form factors $\mathscr{F}(w)$ and $\mathscr{G}(w)$ at the zero recoil point $w=1, \mathscr{F}(1)=\mathscr{G}(1)=1$ [24]. For finite quark masses, the form factors at $w=1$ can be calculated within lattice QCD, Table 4 . 
Table 4: Form factor normalizations at zero recoil $(w=1)$ from lattice QCD.

\begin{tabular}{l|c}
\hline \hline Decay & form factor \\
\hline$B \rightarrow D^{*} \ell v$ & $\mathscr{F}(1)=0.921 \pm 0.013 \pm 0.020$ [25] \\
$B \rightarrow D \ell v$ & $\mathscr{G}(1)=1.074 \pm 0.018 \pm 0.016$ [26] \\
\hline \hline
\end{tabular}

Experiments typically fit the $w$ distribution over almost the entire phase space. The parameterizations of the form factors $\mathscr{F}(w)$ and $\mathscr{G}(w)$ [27] depend on the parameters $\rho_{D^{*}}^{2}, R_{1}(1)$ and $R_{2}(1)$ for $B \rightarrow D^{*} \ell v$, and on $\rho_{D}^{2}$ for $B \rightarrow D \ell v$.

\subsection{Measurements of $B \rightarrow D^{(*)} \ell v$}

Belle has recently shown a preliminary measurement of $B^{0} \rightarrow D^{*-} \ell^{+} v$ using $140 \mathrm{fb}^{-1}$ of $\Upsilon(4 S)$ data [28]. In this analysis the $D^{*+}$ meson and the charged lepton from the signal $B$ decay are reconstructed but no requirement is placed on the second $B$ in the event. In each selected event, decay kinematics constrain the direction of the neutrino momentum to lie on a cone of known opening angle around the $\vec{p}_{D^{*+}}+\vec{p}_{\ell}$ direction. The direction of the missing momentum is used to choose a neutrino direction on this cone.

Once the neutrino momentum is obtained, $w$ of Eq. 4.1 and three decays angles are calculated $(\sigma(w) \approx 0.025)$ and a fit of these four quantities to the $B \rightarrow D^{*} \ell v$ decay width is performed assuming the Caprini et al. parametrization. This allows to extract $\mathscr{F}(1)\left|V_{c b}\right|$ and the quantities $\rho^{2}$, $R_{1}(1)$ and $R_{2}(1)$ of the parameterization. The preliminary results are: $\mathscr{F}(1)\left|V_{c b}\right|=34.4 \pm 0.2 \pm 1.0$, $\rho^{2}=1.293 \pm 0.045 \pm 0.029, R_{1}(1)=1.495 \pm 0.050 \pm 0.062$ and $R_{2}(1)=0.844 \pm 0.034 \pm 0.019$. For all these numbers, the first error is the statistical and the second is the systematic uncertainty. Dominant systematic uncertainties are track reconstruction for $\mathscr{F}(1)\left|V_{c b}\right|$ and background estimation for the form factor parameters.

A measurement of the decays $B \rightarrow D \ell v$ and $B \rightarrow D^{*} \ell v$ using a novel method has recently been published by BaBar [29]: In this analysis, only $D^{0} \ell$ and $D^{+} \ell$ pairs $(l=e, \mu)$ are constructed in $207 \mathrm{fb}^{-1}$ of $\Upsilon(4 S)$ data. The amounts of $B \rightarrow D^{(*)} \ell v$ signal and background in the selected sample are determined using a global fit to three kinematic variables: the c.m. frame momenta of the $D$ meson and of the charged lepton, and $\cos \theta_{D \ell}$. The later is the cosine of the angle between the $D \ell$ and the parent $B$ meson direction in the c.m. frame for signal decays, which can be calculated from event kinematics. The main advantage of this approach is that systematic uncertainties from slow pion reconstruction are completely avoided.

The global fit determines the branching fractions of the decays $B^{+} \rightarrow \bar{D}^{0} \ell^{+} v$ and $B^{+} \rightarrow$ $\bar{D}^{* 0} \ell^{+} v$ and the slopes $\rho_{D}^{2}$ and $\rho_{D^{*}}^{2}$ which enter the Caprini et al. parameterization. The semileptonic decay widths of $B \rightarrow D \ell v$ and $B \rightarrow D^{*} \ell v$ are assumed to be identical for $B^{0}$ and $B^{+}$. These parameters can be converted into the product of $\left|V_{c b}\right|$ times the form factor at zero recoil for $B \rightarrow D \ell v$ decays, $\mathscr{G}(1)\left|V_{c b}\right|=(43.1 \pm 0.8 \pm 2.3) \times 10^{-3}$, and for $B \rightarrow D^{*} \ell v$ decays, $\mathscr{F}(1)\left|V_{c b}\right|=$ $(35.9 \pm 0.2 \pm 1.2) \times 10^{-3}$. The errors are statistical and systematic, respectively.

Finally, BaBar has shown a preliminary measurement of the decay $B \rightarrow D \ell v$ [30]: Using $417 \mathrm{fb}^{-1}$ of $\Upsilon(4 S) \rightarrow B \bar{B}$ events in which the hadronic decay of one $B$ meson is fully reconstructed 

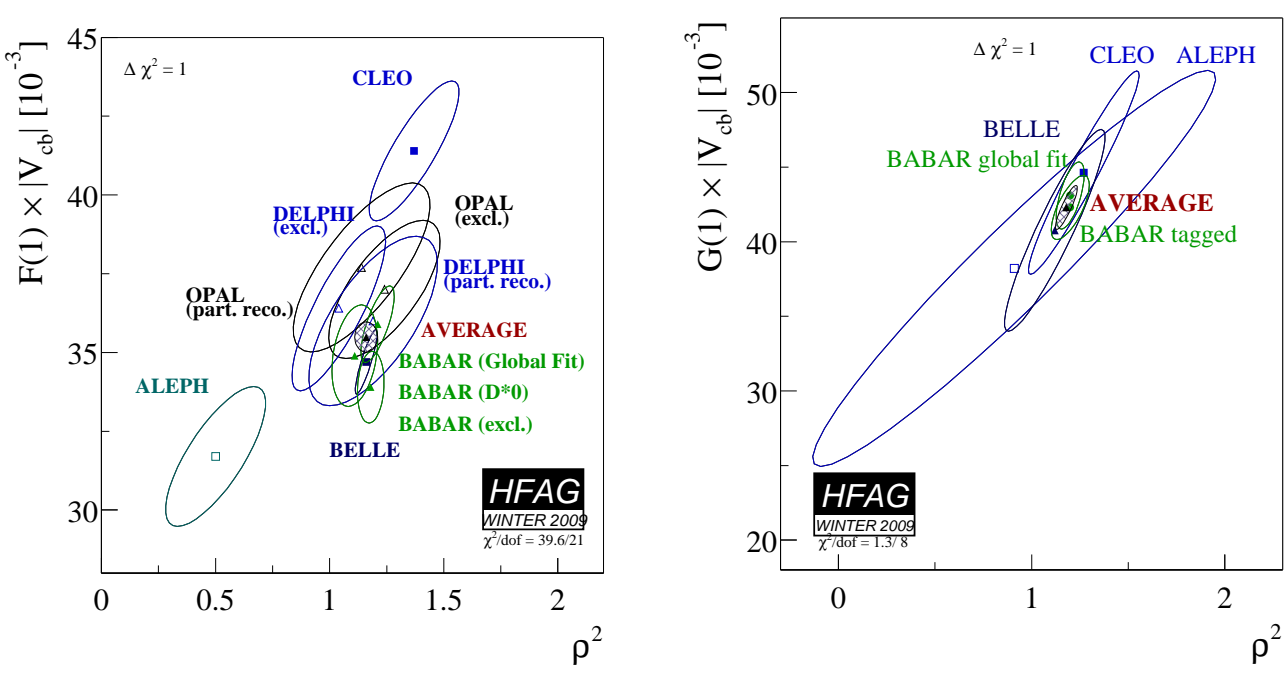

Figure 2: $\Delta \chi^{2}=1$ contours for the HFAG fit to $\left|V_{c b}\right|$ times the form factor at zero recoil and the slope parameter $\rho^{2}$ for $B \rightarrow D^{*} \ell v$ (left) and $B \rightarrow D \ell v$ decays (right).

$\left(B_{\mathrm{tag}}\right)$, semileptonic decays of the other $B$ are selected by identfying a charged lepton and reconstructing either a $D^{0}$ or $D^{+}$meson from the remaining particles in the event. After applying all analysis cuts and subtracting backgrounds $2147 \pm 69 B^{+} \rightarrow \bar{D}^{0} \ell^{+} v$ decays and $1108 \pm 45 B^{0} \rightarrow D^{-} \ell^{+} v$ decays are found.

This tagged analysis minimizes background systematics, which were dominating in previous untagged measurements of $B \rightarrow D \ell v$. Performing a combined fit to both the $B^{+}$and $B^{0}$ samples, $\mathscr{G}(1)\left|V_{c b}\right|$ and the slope $\rho^{2}$ are found to be $(43.0 \pm 1.9 \pm 1.4) \times 10^{-3}$ and $1.20 \pm 0.09 \pm 0.04$ respectively. The stated errors refer to the statistical and systematic ucnertainties.

HFAG has averaged these and older measurements in the $\left(\rho_{D^{*}}^{2}, \mathscr{F}(1)\left|V_{c b}\right|\right)$ and $\left(\rho_{D}^{2}, \mathscr{G}(1)\left|V_{c b}\right|\right)$ planes [31], Fig. 2. For $B \rightarrow D^{*} \ell v$, one obtains $\mathscr{F}(1)\left|V_{c b}\right|=(35.49 \pm 0.48) \times 10^{-3}$ assuming $R_{1}(1)=1.37 \pm 0.064$ and $R_{2}(1)=0.845 \pm 0.038$. For $B \rightarrow D \ell v$, the world average is $\mathscr{G}(1)\left|V_{c b}\right|=$ $(42.3 \pm 1.5) \times 10^{-3}$. While $\chi^{2} /$ ndf. $=1.3 / 8$ for the $B \rightarrow D \ell v$ combination is reasonable, the measurements in the $B \rightarrow D^{*} \ell v$ average are barely consistent $\left(\chi^{2} /\right.$ ndf. $\left.=39.6 / 21\right)$.

\subsection{Summary}

In this article, we have reviewed the status of the determination of the CKM matrix element $\left|V_{c b}\right|$ from both inclusive and exclusive semileptonic $B$ decays to charm. The measurements and combinations presented here are summarized in Table 5, which gives $\left|V_{c b}\right|$ obtained from $B \rightarrow X_{c} \ell v$, $B \rightarrow D^{*} \ell v$ and $B \rightarrow D \ell v$ decays. Note that the $\left|V_{c b}\right|$ numbers given for exclusive decays include a $0.7 \%$ electroweak correction [B2].

The most precise determination of $\left|V_{c b}\right|$ is currently obtained from inclusive decays (1.7\% total uncertainty) followed by $B \rightarrow D^{*} \ell v(2.9 \%)$. There is however a discrepancy of 2.5 standard deviations between these two numbers. 
Table 5: Determination of the CKM matrix element $\left|V_{c b}\right|$ from inclusive semileptonic $B$ decays to charm and from exclusive decays $B \rightarrow D^{(*)} \ell v$.

\begin{tabular}{l|c}
\hline \hline & $\left|V_{c b}\right|\left(10^{-3}\right)$ \\
\hline$B \rightarrow X_{c} \ell v$ inclusive & $41.67 \pm 0.43($ fit $) \pm 0.08\left(\tau_{B}\right) \pm 0.58(t h)$ \\
$B \rightarrow D^{*} \ell v$ exclusive & $38.3 \pm 0.5(\exp ) \pm 1.0(t h)$ \\
$B \rightarrow D \ell v$ exclusive & $39.1 \pm 1.4(\exp ) \pm 0.9(t h)$ \\
\hline \hline
\end{tabular}

\section{References}

[1] N. Cabibbo, Phys. Rev. Lett. 10, 531 (1963);

M. Kobayashi and T. Maskawa, Prog. Theor. Phys. 49, 652 (1973).

[2] F. Tackmann, in these proceedings.

[3] A. Abashian et al. [Belle Collaboration], Nucl. Instrum. Meth. A 479, 117 (2002).

[4] B. Aubert et al. [BABAR Collaboration], Nucl. Instrum. Meth. A 479, 1 (2002).

[5] C. W. Bauer, Z. Ligeti, M. Luke, A. V. Manohar and M. Trott, Phys. Rev. D 70, 094017 (2004).

[6] D. Benson, I. I. Bigi, T. Mannel and N. Uraltsev, Nucl. Phys. B 665, 367 (2003).

[7] P. Gambino and N. Uraltsev, Eur. Phys. J. C 34, 181 (2004).

[8] D. Benson, I. I. Bigi and N. Uraltsev, Nucl. Phys. B 710, 371 (2005).

[9] http://www.slac.stanford.edu/xorg/hfag/semi/index.html

[10] B. Aubert et al. [BABAR Collaboration], Phys. Rev. D 69, 111103 (2004).

[11] B. Aubert et al. [BABAR Collaboration], arXiv:0707.2670 [hep-ex].

[12] B. Aubert et al. [BABAR Collaboration], Phys. Rev. D 69, 111104 (2004).

[13] B. Aubert et al. [BABAR Collaboration], Phys. Rev. D 72, 052004 (2005).

[14] B. Aubert et al. [BaBar Collaboration], Phys. Rev. Lett. 97, 171803 (2006).

[15] C. Schwanda et al. [BELLE Collaboration], Phys. Rev. D 75, 032005 (2007).

[16] P. Urquijo et al. [BELLE Collaboration], Phys. Rev. D 75, 032001 (2007).

[17] K. Abe et al. [Belle Collaboration], arXiv:0804.1580 [hep-ex].

[18] D. E. Acosta et al. [CDF Collaboration], Phys. Rev. D 71, 051103 (2005).

[19] S. E. Csorna et al. [CLEO Collaboration], Phys. Rev. D 70, 032002 (2004).

[20] S. Chen et al. [CLEO Collaboration], Phys. Rev. Lett. 87, 251807 (2001).

[21] J. Abdallah et al. [DELPHI Collaboration], Eur. Phys. J. C 45, 35 (2006).

[22] A. Hocker and V. Kartvelishvili, Nucl. Instrum. Meth. A 372, 469 (1996).

[23] C. Schwanda et al. [Belle Collaboration], Phys. Rev. D 78, 032016 (2008).

[24] M. Neubert, Phys. Rept. 245, 259 (1994). 
[25] C. Bernard et al., Phys. Rev. D 79, 014506 (2009) [arXiv:0808.2519 [hep-lat]].

[26] M. Okamoto et al., Nucl. Phys. Proc. Suppl. 140, 461 (2005) [arXiv:hep-lat/0409116].

[27] I. Caprini, L. Lellouch and M. Neubert, Nucl. Phys. B 530, 153 (1998).

[28] I. Adachi et al. [Belle Collaboration], arXiv:0810.1657 [hep-ex].

[29] B. Aubert et al. [BABAR Collaboration], Phys. Rev. D 79, 012002 (2009) [arXiv:0809.0828 [hep-ex]].

[30] B. Aubert et al. [BABAR Collaboration], arXiv:0807.4978 [hep-ex].

[31] The new Belle $B^{0} \rightarrow D^{*-} \ell^{+} v$ measurement [28] is not yet included in the HFAG winter 2009 average.

[32] A. Sirlin, Nucl. Phys. B 196, 83 (1982). 